\title{
Artificial Intelligence in Materials Science
}

\author{
Matthew N. O. Sadiku, Guddi K. Suman, and Sarhan M. Musa \\ Roy G. Perry College of Engineering \\ Prairie View A\&M University \\ Prairie View, TX 77446
}

\begin{abstract}
Artificial intelligence (AI) is changing the way we discover new materials. The emergence of AI brings a new dawn to the development of material science. It enables material researchers to harness the power of machine learning and artificial intelligence to develop a system that autonomously discovers new materials. AI is also helping materials scientists and engineers to revolutionize the way of understanding and discovering materials used in applications ranging from aerospace engineering to soft robotic prosthetics. This paper provides an introduction to the uses of AI in materials science.
\end{abstract}

Key Words: Artificial Intelligence, Machine Learning, Materials, Research.

\section{INTRODUCTION}

Materials have been key to the growth, prosperity, security, and life quality of humans. Material science mainly aims at exploring the relationship between materials structure, process, properties, and application. Over the past few years, materials science has undergone a rapid transformation facilitated by the pervasive data revolution. Traditional materials science research is a time-consuming, human-centered process that takes a certain kind of individual with the knowledge, patience, and understanding to design, conduct, analyze, and interpret experimental data.

Materials scientists are often seeking for materials that have superior properties. They characterize and measure the properties of materials using three major tools: analytical, experimental, and computer simulation [1]. Scientific discovery evolves from the "experimental" through the "analytical" and "computational." Experiments have traditionally been used to play a key role in characterizing or designing new materials. Simulation is essentially computational modeling. A combination of experimentation and computer simulations has contributed towards exploring materials design more economically [2]. It has allowed researchers to cut substantially the time and cost of materials design.

One of the most exciting tools that have entered the material science toolbox is artificial intelligence. The emergence of artificial intelligence (AI) brings a new dawn to the development of material science. The main purpose of AI is to aid human capabilities and help us make advanced decisions with far-reaching consequences. There has been an ever-increasing application of machine learning (ML) and artificial intelligence (AI) techniques by materials researchers. 


\section{REVIEW ON ARTIFICIAL INTELLIGENCE}

Artificial intelligence (AI) refers to computer systems that mimic human cognitive functions. It is a field of computer science that deals with intelligent machines. AI has a long history that is actively and constantly changing and growing. The term "artificial intelligence" was first used at a Dartmouth College conference in 1956. The main goal of AI is to enable machines to perform complex tasks that typically require human intelligence $[3,4]$. In simple terms, AI attempts to clone human behavior. An important feature of AI technology is that it can be added to existing technologies. AI is now one of the most important global issues of the $21^{\text {st }}$ century. It is poised to disrupt our world and change processes and developments in science, engineering, education, business, entertainment, and agriculture.

The concept of AI is an umbrella term that encompasses many different technologies. AI is not a single technology but a collection of techniques that enables computer systems to perform tasks that would otherwise require human intelligence [5]. The major disciplines in AI include:

- Expert systems

- Fuzzy logic

- Neural networks

- Machine learning $(M L)$

- Deep learning

- Natural Language Processors (NLP)

- Robots

These AI tools are illustrated in Figure 1. Each AI tool has its own advantages. Using a combination of these models rather than a single model is recommended. AI systems are designed to make decisions using real-time data. They have the ability to learn and adapt as they make decisions.

AI has benefited many areas such as chemistry and medicine, where routine diagnoses can be initiated by AI-aided computers. It embraces a wide range of disciplines such as computer science, engineering, machine learning, chemistry, biology, physics, astronomy, medicine, neuroscience, social sciences, and the military. Today, AI algorithms are integrated into our daily lives in several forms, such as personal assistants, automated mass transportation, aviation, computer gaming, facial recognition at passport control, voice recognition on virtual assistants, driverless cars, companion robots, wearables, etc. [6]. AI algorithms are increasingly used to open up new horizons for scientists and engineers.

\section{A.I. IN MATERIALS SCIENCE}

Modern civilization heavily depends on technology. Materials have always been technology enablers, and currently, there are many key technology areas that await materials discovery and processing solutions. Addressing these opportunities will drive and propel the required developments. Today, we can harness the power of digital technologies to accelerate the discovery of new materials by marrying AI and materials science. The marriage of artificial intelligence and materials science represents one of the examples of the fourth industrial revolution. Computational materials science has made significant progress since the concept of materials science in the 1950s.

Traditionally, materials scientists have been using a conventional experimental process to identify and discover new composite materials from scratch. They design a new material, make it, and then test it, but this approach is time-consuming and subject to error. The development of new materials is painfully slow. 
AI, when applied to material science, offers the possibility of speeding up the materials-discovery process. Figure 2 illustrates the application of AI in material science [7]. Machine learning, a branch of AI, gives us a tool to search a large amount of space in little time. It often requires input on what to look for, where to look, and data that the machine can learn from.

\section{APPLICATIONS}

There are various applications of $\mathrm{AI}$ and $\mathrm{ML}$ in materials science. Current applications of $\mathrm{AI}$ in materials science focus more on solving engineering and design problems than on directly deriving fundamental scientific insight from data. A FAIR treatment encompasses the four principles: findability, accessibility, interoperability, and repurposability. The FAIR principles can guide the materials science and engineering community in developing infrastructure suited to collaboration [8].

Some companies are capitalizing on using AI to develop advanced materials.

Citrine Informatics has developed AI software to narrow the choice of materials to test for a given application. Zymergenis combines AI and automation in biology and materials science to improve industrial microbes.

\section{BENEFITS}

We are currently experiencing an explosion of works that develop and apply AI to materials. Advances in AI for materials research are as important as ever for accelerating innovation in materials. Artificial intelligence can help us more efficiently and effectively practice scientific inquiry. It could help promote the development of material science and accelerate the invention of new materials. Through the use of ML, AI could outperform many material scientists and make a more efficient prediction of new materials. AI is employed in modern materials research to develop new technologies. AI could reduce the time needed to develop a new material to one-two years from the current 10-20 years [9].

\section{CHALLENGES}

$\mathrm{AI}$ and machine learning face some challenges and limitations machine in their application to materials science. To expand the application of AI methods and realize the full potential of AI, the materials community must develop AI systems that can represent, evaluate, and perform inference about physical mechanisms underlying observational data. Current applications of AI in materials science largely ignore the uncertainty of the raw data used to train models [10].

One of the most significant concerns about deep learning in the scientific community right now is regarding the accuracies of the results. In particular, there is no consensus on what is the minimum amount of data required for a given property prediction.

For sure, AI will not completely replace human at the work of material research but will serve as a powerful tool to accelerate the progress of materials discovery. Material researchers will need to learn to master AI tools to decrease the trial error times, solve more difficult material problems [11]. 


\section{CONCLUSION}

Artificial intelligence is a branch of computer science with the ambitious goal of studying and designing intelligent systems. It has raised the expectation that AI can revolutionize scientific discovery in general and materials science specifically. AI is also impacting materials science by employing machine learning algorithms to automate specific research tasks. The scientific community is currently exploring the use of AI for scientific discovery, among other things [12].

There is a critical need for workforce development to ensure that AI techniques are introduced into the materials science workflow. It is therefore expedient to educate the next generation workforce to be conversant in AI techniques. Materials science curricula need urgent restructuring to produce a competitive next-generation workforce. More information on the uses of AI in material science can be found in the books in [13,14] and related journal: Materials Letters.

\section{REFERENCES}

[1] M. N. O. Sadiku, Computational Electromagnetics with MATLAB. Boca Raton: CRC Press, 4th ed., 2019, p. 1.

[2] R. Batra, "Accurate machine learning in materials science facilitated by using diverse data sources," Nature, January 2021, pp. 524-525.

[3] M. N. O. Sadiku, "Artificial intelligence", IEEE Potentials, May 1989, pp. 35-39.

[4] S. Greengard, "What is artificial intelligence?" May 2019, https://www.datamation.com/artificial-intelligence/what-is-artificial-intelligence.html

[5] https://in.pinterest.com/pin/828662400161409072/

[6] Y. Mintz and R. Brodie, "Introduction to artificial intelligence in medicine," Minimally Invasive Therapy \& Allied Technologies, vol. 28, no. 2, 2019, pp. 73-81

[7] M. Todorovic, "Artificial intelligence for materials research," https://mycourses.aalto.fi/course/view.php?id=15280

[8] B. L. DeCost, et al., "Scientific AI in materials science: A path to a sustainable and scalable paradigm," Machine Learning: Science and Technology, vol. 1, no. 3, July 2020.

[9] M. Annunziata, "Mind over matter: Artificial intelligence can slash the time needed to develop new materials," December 2018, https://www.forbes.com/sites/marcoannunziata/2018/12/03/mind-over-matter-artificial-intelligence-and-materialsscience/?sh $=3 \mathrm{~b} 088153 \mathrm{e} 9 \mathrm{db}$

[10] J. Schmidt et al., "Recent advances and applications of machine learning in solid-state materials science," npj Computational Materials, vol. 5, no. 83, 2019.

[11] W. Sha, et al., "Artificial intelligence to power the future of materials science and engineering," Advanced Intelligent System, February 2020

[12] C. P. Gomes, B. Selman, and J. M. Gregoire, "Artificial intelligence for materials discovery,” MRS Bulletin, vol. 44, 2019, pp. 538-544.

[13] Y. Cheng, T. Wang and G. Zhang (eds.), Artificial Intelligence for Materials Science. Springer 2021.

[14] R. J. Harrison and L. D. Roth (eds.), Artificial Intelligence Applications in Materials Science: Proceedings. Tms, 1987

\section{ABOUT THE AUTHORS}

Matthew N.O. Sadiku is a professor emeritus in the Department of Electrical and Computer Engineering at Prairie View A\&M University, Prairie View, Texas. He is the author of several books and papers. His areas of research interest include computational electromagnetics and computer networks. He is a fellow of IEEE.

Guddi K. Suman is currently working towards a Ph.D. in Electrical and Computer Engineering at Prairie View A\&M University, Prairie View, TX. Her areas of research interest include space radiation effects on electronics, nanomaterials, characterization of nanomaterials and semiconductors, thin film nanofabrication, nanosensors, and AI in material science. Currently, she worked as an intern at Pacific Northwest National Laboratory, Richland, WA in 2021. She is a member of IEEE.

Sarhan M. Musa is a professor in the Department of Electrical and Computer Engineering at Prairie View A\&M University, Texas. He has been the director of Prairie View Networking Academy, Texas, since 2004. He is an LTD Sprint and Boeing Welliver Fellow. His areas of research interest include computational electromagnetics and computer networks. 


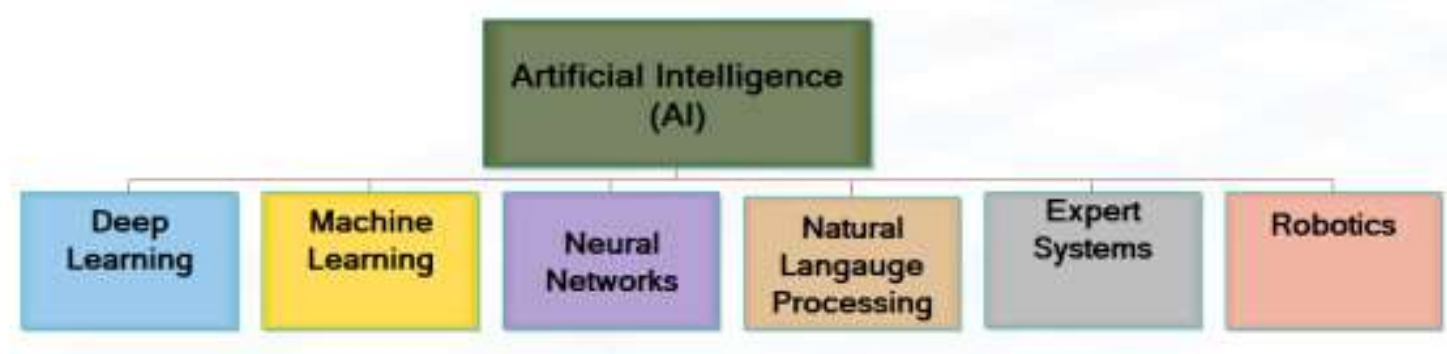

Figure 1 Different branches of artificial intelligence.

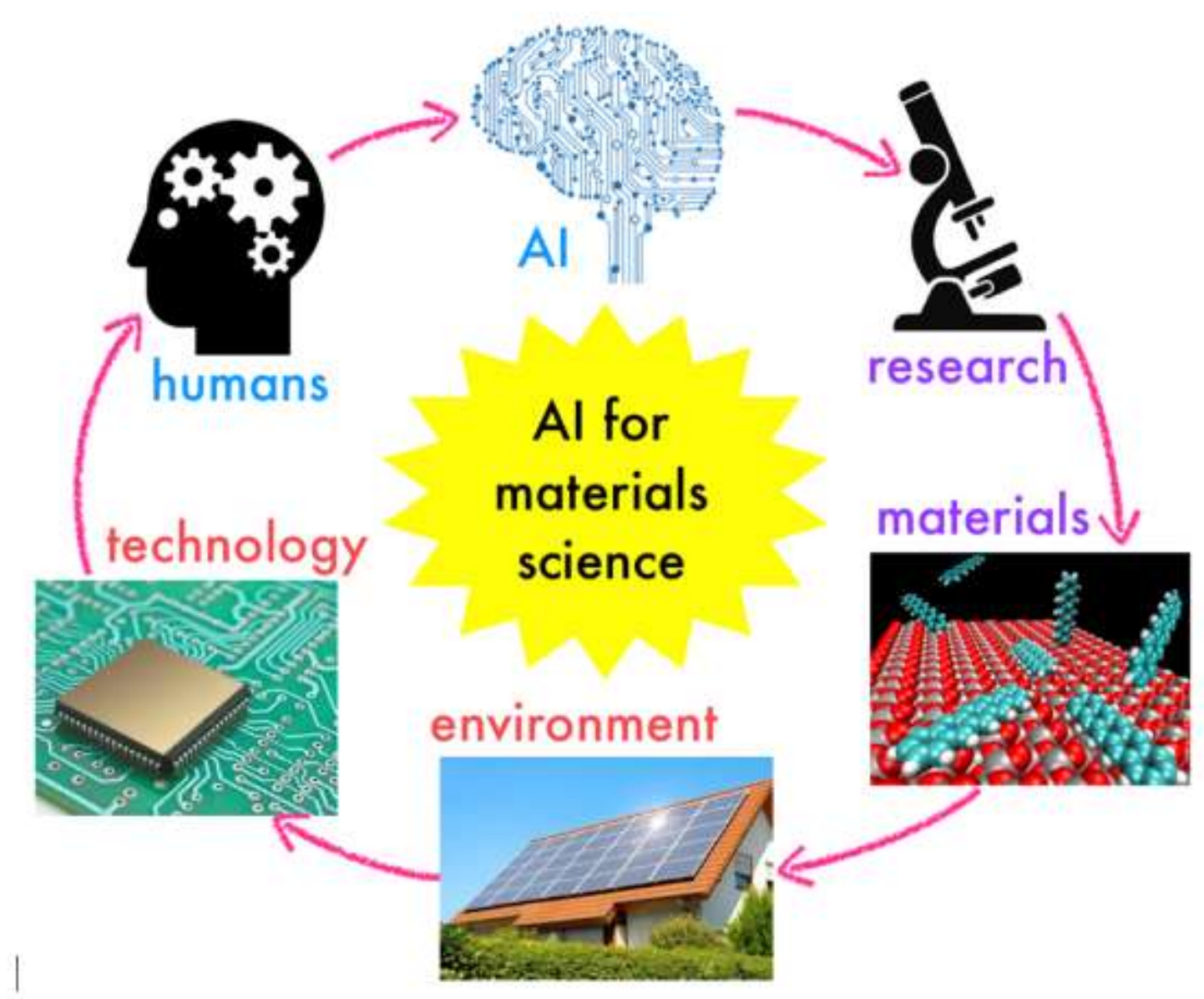

Figure 2 Artificial intelligence for material science [7]. 